\title{
WHAT SHOULD A DENTIST BE AWARE OF CONCERNING SYMPTOMS OF SLEEP DISORDERS IN THE ORAL CAVITY?
}

\author{
Joanna Smardz, Wojciech Florjanski, Sylwia Orzeszek, Andrzej Malysa, Marek Zietek, Mieszko Wieckiewicz \\ Department of Experimental Dentistry, Wroclaw Medical University, Wroclaw, Poland
}

\begin{abstract}
Sleep bruxism (SB) is a common and complex phenomenon that can result in serious clinical consequences concerning the stomatognathic system including damage to dental hard tissues, especially tooth wear, damage to prosthetic restorations, damage to the periodontium and oral mucosa, and a spectrum of symptoms affecting masticatory muscles. However, there are other conditions with similar clinical manifestations that may resemble or even in some cases mimic SB. The aim of this study was to evaluate the most common sleep-related conditions that can cause clinical implications similar to SB and to emphasize the methods of carrying out a proper differential diagnosis. PubMed, Web of Science, and Scopus databases were searched for current literature on the manifestation of selected sleep disorders in the stomatognathic system. Forty-one articles were finally included in this review. Conditions such as oromandibular myoclonus, dystonia, Parkinson's disease and parkinsonism, epileptic and non-epileptic motor phenomena were included in this review as potentially mimicking the symptoms of sleep bruxism. Included studies indicate that the combination of accurate medical history interview, thorough clinical examination and video-polysomnography seems to be the key to proper differential diagnostics between SB and other medical conditions that can occur during sleep and present similar clinical symptoms affecting the stomatognathic system.
\end{abstract}

KEY WORDS: sleep disorders, tooth wear, polysomnography, sleep bruxism.

J Stoma 2019; 72, 4: 172-178

DOI: https://doi.org/10.5114/jos.2019.91235

\section{INTRODUCTION}

Tooth wear is an irreversible process resulting in loss of mineralized tooth substance (enamel and dentine) [41]. It can occur due to mechanisms of attrition (caused by direct contact between teeth situated in opposite dental arches), abrasion (caused by contact of teeth and extrinsic objects) and erosion (by dissolution of teeth structure by acids without any involvement of bacteria); some authors also add abfraction (cervical loss of enamel, not caused by caries but by excessive occlusal stress) [2, 31, 33]. Many of the recent published studies show that tooth wear has become a common and globally increasing problem in the everyday practice of general dentists $[27,40]$. The patients affected by tooth wear may suffer from adverse change of facial and smile appearance, hypersensitivity, decrease of chewing and biting efficiency [27]. Diagnosing the causes of tooth wear is in many cases challenging, due to multifactorial etiology, especially taking in account that more than one mechanism of tooth wear can act at the same time [33]. Proper diagnosis becomes even more difficult considering sleep-related conditions leading to tooth wear. Due to clinicians' scarce knowledge regarding these conditions, unreliable information from self-report, and unspecific clinical symptoms,
JOURNAL OF STOMATOLOGY CZASOPISMO STOMATOLOGICZNE
ADDRESS FOR CORRESPONDENCE: Joanna Smardz, Department of Experimental Dentistry, Wroclaw Medical University, 26 Krakowska St., 50-425 Wroclaw, Poland, e-mail: joannasmardz1@gmail.com

ReCEIVED: 18.11.2019 • ACCEPTED: 25.11.2019 • Published: 12.12.2019 
many clinicians simply attribute pathological tooth wear to sleep bruxism (SB) [33]. Furthermore, SB is a complex phenomenon that can result in other serious clinical consequences concerning the masticatory system such as damage to dental hard tissues other than tooth wear, damage to the periodontium and oral mucosa, and a spectrum of symptoms affecting masticatory muscles. In fact, there are other conditions with similar clinical manifestations that may resemble or even in some cases mimic SB. In this article we would like to review the most common of these conditions, emphasizing the methods of carrying out differential diagnosis and basic dental implications.

\section{OBJECTIVES}

The aim of this study was to evaluate the most common sleep-related conditions that can cause clinical implications similar to SB and to emphasize the methods of carrying out a proper differential diagnosis.

\section{MATERIAL AND METHODS}

PubMed, Web of Science and Scopus databases were searched for current literature on the manifestation of selected sleep disorders in the oral cavity. The majority of articles were in English, but there was also one Spanish article included during the preparation of this paper. The analysis included original articles, randomized clinical trials, case-control studies and literature reviews published until September 2019.

\section{RESULTS}

There were 41 articles included in this review. Among the qualified studies 11 concerned the subject of bruxism, 9 addressed the problem of patients with dystonia, 5 concerned Parkinson's disease or parkinsonism and 2 concerned myoclonus. The authors also included 9 papers that concerned epileptic and non-epileptic motor phenomena. Observation of symptoms in patients' oral cavities similar to those found in patients with bruxism was made.

\section{SLEEP BRUXISM}

Sleep bruxism (SB) is currently defined as an activity of masticatory muscles during sleep that can be phasic (contractions lasting 0.25-2 s) or tonic (contractions lasting more than $2 \mathrm{~s}$ ) or mixed and is not considered as a movement disorder in otherwise healthy individuals $[5,21,35]$. The prevalence of SB varies from 8 to $31 \%$ of the adult population [35]. SB occurs most often in non-rapid eye movement 1 (N1) and non-rapid eye movement 2 (N2) sleep stages (90\%), less often in rapid eye movement (REM) sleep $(10 \%)[5,41]$. The etiology of this phenomenon is complex and unclear. It is believed that genetic (especially regarding neurotransmitters and arousals), psychological (personality traits, stress sensitivity and anxiety) and exogenous factors (nicotine, caffeine, alcohol and drug use) may play a role in bruxism occurrence and severity $[5,21,26]$.

SB can manifest in different forms involving tooth contact (clenching and grinding), and without tooth contact: bracing and thrusting of the mandible. Only the first two forms may be considered as risk factors for tooth wear. It is hard to define cut-off points dividing clinically irrelevant SB from SB causing negative health implications [23]. SB is a common phenomenon that is often associated with tooth wear and has up to now been within the diagnostic and therapeutic interest of dentists. There are plenty of symptoms concerning the masticatory system that can be associated with SB. The most common are: the spectrum of damage of dental hard tissues and prosthetic restorations, oral mucosa injuries and implications connected with masticatory muscles such as hypertrophy, increased tension and pain [42]. Unfortunately, none of those symptoms are specific to SB. The study conducted by Manfredini et al. on 41 healthy volunteers showed that tooth wear cannot be an indicator of ongoing SB due to the lack of correlation between sleep-time muscle activity and tooth wear [24]. Also SB cannot be considered a direct cause of muscle pain as some studies report [9, 34]. In light of these facts, diagnosis based on self-report and clinical examination turns out to be insufficient. That is why the current gold standard in diagnosing SB and for differential diagnosis is polysomnography [28]. This method combines electromyography, electroencephalography and audio-visual recordings of sleeping individuals, giving a wide range of accurate information [8]. The method is proven to be more accurate than selfreport or even third party report in SB [28].

SB is currently managed by occlusal splints, psychological and pharmacological therapies or biofeedback [26].

\section{OROMANDIBULAR MYOCLONUS}

Myoclonus involves sudden, fast and involuntary movements lasting 10-50 ms, sometimes over $100 \mathrm{~ms}$. They are caused by short muscle spasms (positive myoclonus) or by a sudden short break in muscle tension (with its relaxation and loss of bioelectrical activity) that occurs during tonic muscle contraction (negative myoclonus). Examples of physiological myoclonus include: jerks during sleep (hypnic jerks), exercise-induced myoclonus, hiccups, and reflex myoclonus caused by a strong external stimulus such as noise, light or pain [19].

Depending on the origin of the myoclonus, cortical, subcortical, extrapyramidal, trunk, cerebellar, spinal and peripheral are distinguished. Myoclonic jerks are very often incorrectly classified as sleep bruxism events. 
It is estimated that approximately $10 \%$ of patients clinically diagnosed with SB are in fact suffering from oromandibular myoclonus (OMM) and in some patients both of these phenomena coexist. Similarly to bruxism, OMM occur most frequently in N1 and N2 sleep stages, rarely in REM sleep. In the orofacial area, they usually take the form of one or two short contractions, shorter than the episodes of bruxism $(<0.25 \mathrm{~s})$, usually 1 up to 2 bursts, more rarely cluster-type bursts with 3 or even more contractions with intervals less than $3 \mathrm{~s}[1,19]$. Symptoms of myoclonus in the orofacial region include: destruction of dental hard tissues and oral mucosa, abnormal tooth mobility, and pain in the temporomandibular region. Tooth wear can be similar to that found in people with sleep bruxism. The treatment of myoclonus differs from the bruxism management process, which is why differential diagnosis of these two phenomena is so important [19]. The diagnosis based solely on the clinical examination and symptoms reported by patients may turn out to be incorrect, because symptoms which we can observe in the oral cavity in patients with SB can also appear in other movement disorders. That is why polysomnographic examination is so important in the process of differential diagnosis [1].

\section{DYSTONIA}

Dystonia is a movement disorder leading to similar damage in tooth hard tissues as that caused by bruxism. Dystonia is a disorder of involuntary, repetitive, predominantly sustained (tonic), or spasmodic (rapid or clonic resembling myoclonus) muscle contractions [17]. Dystonia may affect almost all striated muscle groups in different body regions. For the dentist, craniocervical dystonia, in particular orofacial dystonia (OFD) and oromandibular dystonia (OMD) are important [15]. OMD is a rare neurological disorder, which in the majority of cases affects women [15]. OMD may occur alone or as a part of a segmental or generalized dystonia and may be idiopathic or secondary to previous medication such as neurological agents or drug use, trauma such as traumatic brain injury, stroke, brain tumor, or neurodegenerative diseases $[7,15]$. In addition, cases of dystonia due to local trauma or lesions associated with reconstructions of occlusion in dental patients have been reported [15]. Dystonia may exhibit spontaneous remission, usually within 5 years, but such cases are rare. Oromandibular dystonia is further subdivided into: jaw opening, jaw closing, jaw deviating and lingual dystonia $[6,32]$.

OFD presents as involuntary, persistent contractions of the periorbital muscles, face, mouth and jaw, throat, larynx or cervix. It most frequently affects masticatory muscles and tongue muscles. Pathological muscle spasms can cause jaw closing, jaw opening, jaw deviation, facial grimacing, tongue movements, lip pursing, mouth-closing disturbance, tooth wear/loss, or damage to the oral soft tissues. Furthermore, it may also lead to dysphagia, dysarthria, difficulties in breathing, weight loss secondary to mechanical problems with food intake, temporomandibular disorders, pain, and impaired sleep quality, or a combination of these symptoms [11, 12, 32, 39]. Dystonia can also cause depression and anxiety [6].

Patients suffering from dystonia report the feeling of discomfort in the face and jaws as well as fatigue and tension in the affected muscles. An increase in dystonic activity is commonly observed during eating and stress with activity reduction during rest and sleep $[6,15]$.

Dystonia is primarily a clinical diagnosis based on the history and phenomenology, with an electromyographic examination supporting the diagnosis and magnetic resonance imaging of the brain and spinal cord used mainly when secondary dystonia due to CNS lesion is suspected. Dystonia rating scales are also available, but their clinical utility is questionable [15]. Due to diagnostic difficulties, the manifestation of oral dystonia is often incorrectly diagnosed as a dental problem, bruxism or temporomandibular disorder $[6,12,15]$.

Management of OMD includes physical and speech therapy, massage, biofeedback, acupuncture, occlusal appliances, pharmacotherapy, botulinum toxin injections and surgery [15].

\section{PARKINSON'S DISEASE AND PARKINSONISM}

Parkinson's disease is a currently irreversible extrapyramidal disorder with incompletely clear etiology. It is the most common cause of idiopathic parkinsonism a broader term describing a group of movement disorders characterized by bradykinesia, hypokinesia, tremor at rest, muscle rigidity and postural disturbances that may affect the tongue and lips, which in turn affects oral functions such as chewing, swallowing and talking and can cause pathological abrasion of teeth [22]. An important dental aspect in parkinsonism is the limitation of saliva flow, which results in xerostomia, greater soft tissue susceptibility to injuries and a higher incidence of carious lesions. Vorheff and Lobezzo conducted a study in which patients with Parkinson's disease or other parkinsonism reported pain in the temporomandibular region (TMD) and bruxism statistically significantly more often than healthy controls [38]. Parkinson's disease is significantly associated with an increased risk of TMD and in consequence with pathological tooth wear [10]. Also, chronic l-dopa therapy commonly used in Parkinson's disease management can induce levodopa-induced dyskinesia (LID). It presents typically as choreiform movements and involves all parts of the body, especially the head and neck $[3,36]$.

\section{EPILEPTIC AND NON-EPILEPTIC MOTOR PHENOMENA}

Among the noteworthy phenomena that also may result in the damage of dental hard tissues and temporo- 
mandibular disorders are sleep induced seizures [13, 14]. Phenomena that may be the most interesting in this aspect are nocturnal frontal lobe seizures (NFLS) and non-epileptic paroxysmal (NES) events in sleep due to diagnostic difficulties that occur even during video-polysomnography.

Non-epileptic seizures are paroxysmal events that look very similar to epileptic seizures. These events do not involve abnormal, rhythmic discharges of cortical neurons. Usually they are caused by either psychological or physiological conditions $[16,18,20,25]$. That is why nonepileptic seizures (NES) can be divided into two subtypes: organic non-epileptic seizures and psychogenic seizures. A newer name for non-epileptic seizures is "dissociative seizures". This is helpful because it does not describe seizures in terms of epilepsy [16, 18, 29]. From a dental point of view, treatment should be limited to damaged teeth tissues restoration, because removal of the causative disease of NES causes seizures to cease.

In turn, nocturnal frontal lobe epilepsy (NFLE) is a condition characterized primarily by seizures occurring exclusively or mainly during sleep, originating from the mesial frontal or orbitofrontal regions [30]. They frequently have unusual or frankly bizarre ictal semiology and tend not to show pathology on MRI brain scan and interictal or even ictal EEG [30]. Ryvlin et al. believe that this is a mild epileptic syndrome, with a minimal neuropsychological impact [30]. At the same time, they state that no study has evaluated the neuropsychological profile of patients suffering from NFLE [30, 37].

Only some of the studies noted the emerging problem of increased tooth wear and the impact of the above medical conditions on temporomandibular region. The same mentions were highly marginalized $[13,14$, $20]$. If needed, dental treatment should be based on protecting teeth from damage. The current state of the art provides considerable scope for targeted and thorough research in the future.

Clinicians usually base their diagnosis on the patient's self-report and clinical examination; only those methods are possible and probable bruxism can be determined.

\section{DISCUSSION}

Damage to dental hard tissues is the most common problem associated with bruxism. Usually the dentists are the first to be informed about this issue $[4,5,21]$. To establish the cause of the condition, self-report and clinical examination are most commonly used. Unfortunately, such tools only allow one to determine the presence of possible or probable SB and are insufficient to diagnose definite bruxism. To diagnose definite SB and for correct differential diagnosis instrumental methods are necessary [21]. Among them, the most accurate examination is audio-video-polysomnography. Electromyography, which is part of the polysomnographic examination, allows for accurate classification of SB episodes as phasic lasting $0.25-2 \mathrm{~s}$, tonic lasting above $2 \mathrm{~s}$ and mixed [5]. Video recording allows observation of the mandible movements. Audio recording allows one to listen to the grinding sounds. Although symptoms such as damage of dental hard tissues, prosthetic restorations and oral mucosa are common and often accompany SB, similar symptoms can be associated with other sleep conditions. Most often similar symptoms are caused by OMM. Also, OMM episodes may be similar to $\mathrm{SB}$ episodes and mimic them. What is more, OMM episodes occur in N1 and N2 sleep stages, less in REM sleep, i.e. similarly as SB episodes. Both types of episodes can also occur in the same patient simultaneously and independently. The key to observe the difference between OMM and SB in polysomnographic examination is electromyographic recording. OMM episodes are shorter and usually last 25-50 ms, less often $100 \mathrm{~ms}$ [1, 19]. Also, therapeutic pathways of these two phenomena are partially different, which indicates the importance of proper differential diagnosis. Due to the fact that OMM and SB occur during sleep, i.e. outside of the patient's consciousness, their presence may remain unnoticed for a long time and the first symptoms observed may be visible in the oral cavity.

The second most important disorder in terms of SB differential diagnosis is dystonia, especially OFD and OMD [15]. Similarly to SB, dystonia can cause a spectrum of damage of dental hard tissues and prosthetic restorations and oral mucosa and increase the muscle implication of TMD risk. However, additional symptoms may be seen in dystonia that are not usually associated with SB. These include dysphagia, dysarthria, breathing difficulties and disturbances in jaw movements $[6,11,12$, 32, 39]. Dystonia episodes can be observed as involuntary, repetitive, sustained (tonic) or spasmodic (rapid or clonic) muscle contractions [20]. Therapeutic pathways of these two phenomena are partially different, which indicates the importance of proper differential diagnosis. Differential diagnostics include thorough medical history interview, electromyography or polysomnography and sometimes magnetic resonance imaging.

There are also other general conditions that can result in symptoms within the stomatognathic system. These include Parkinson's disease or epileptic and non-epileptic motor phenomena. All of these disorders can also present night episodes and give symptoms similar to SB. In the case of these diseases, firstly, a thorough medical history interview will play a very important diagnostic role, because symptoms associated with the stomatognathic system are not the first to appear in these diseases and in most cases the patient is aware of the disease. Both Parkinson's disease and epileptic and non-epileptic motor phenomena need neurological assessment.

Summary of study findings is presented in Table 1. 


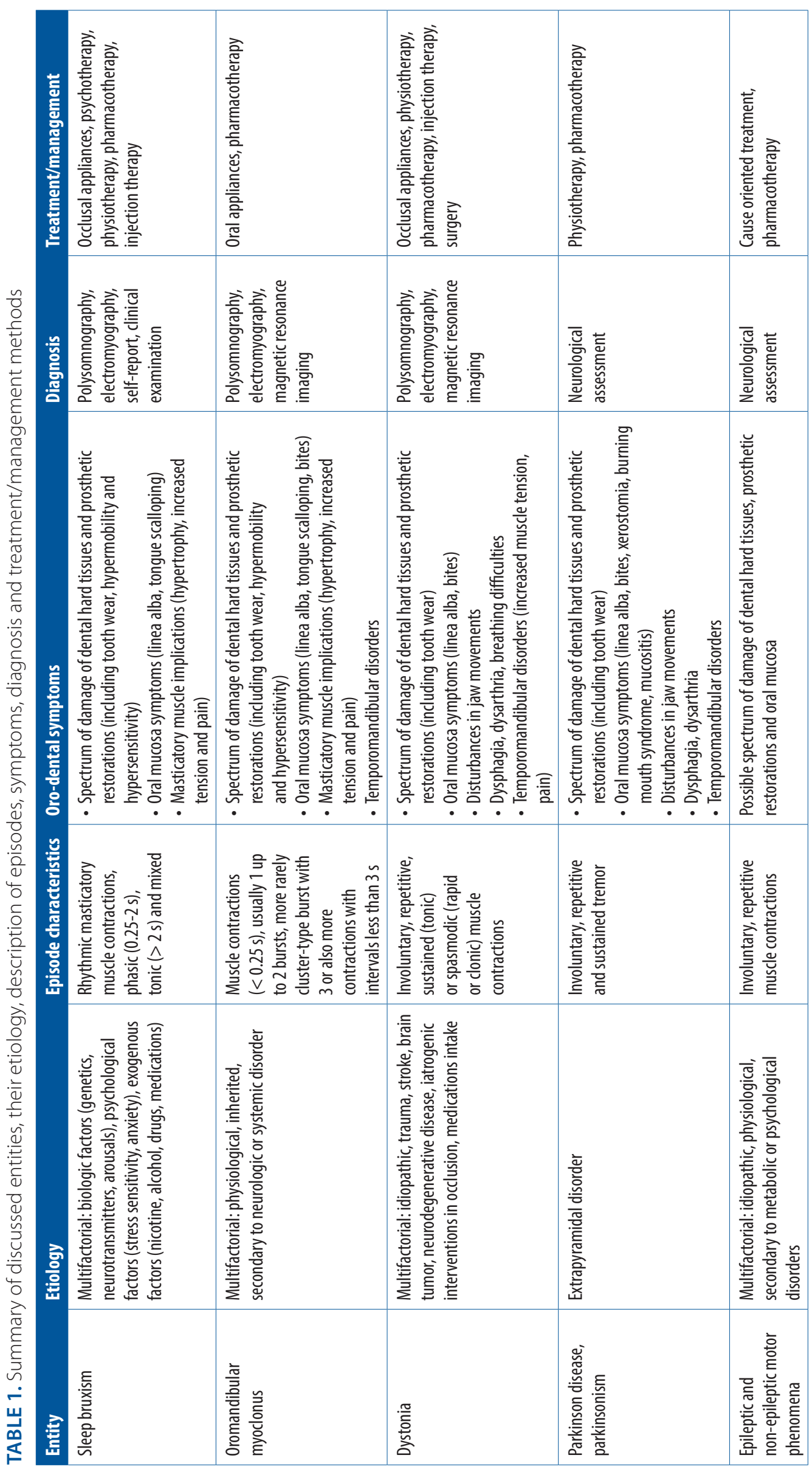




\section{CONCLUSIONS}

There are numerous disorders occurring during sleep that can have very similar clinical implications for the stomatognathic system as SB. This indicates the absolute need for an accurate diagnosis and differentiation of SB from disorders with sleep-related events. The combination of accurate medical history interview, thorough clinical examination and audio-video-polysomnography seems to be the key to proper differential diagnostics between SB and other medical conditions that can occur during sleep and present similar clinical symptoms affecting the stomatognathic system.

\section{CONFLICT OF INTEREST}

The authors declare no potential conflicts of interest with respect to the research, authorship and/or publication of this article.

\section{References}

1. Abe S, Gagnon JF, Montplaisir JY, et al. Sleep bruxism and oromandibular myoclonus in rapid eye movement sleep behavior disorder: a preliminary report. Sleep Med 2013; 14: 1024-1030.

2. Addy M, Shellis RP. Interaction between attrition, abrasion and erosion in tooth wear. Monogr Oral Sci 2006, 20: 17-31.

3. Agarwal PA. Severe jaw-opening dystonia as an unual manifestation of levodopa-related wearing-off in Parkinson's disease, and successful treatment with botulinum toxin injection. Mov Disord Clin Pract 2019; 6: 500-501.

4. Al-Omiri M, Lamey PJ, et al. Impact of tooth wear on daily living. Int J Prosthodont 2006; 19: 601-605.

5. American Academy of Sleep Medicine. International Classification of Sleep Disorders, 3rd ed. Darien, IL, USA: American Academy of Sleep Medicine; 2014.

6. Bakke M, Baram S, Dalager T, Biernat HB, Møller E. Oromandibular dystonia, mental distress and oro-facial dysfunction - a follow-up 8-10 years after start of treatment with botulinum toxin. J Oral Rehabil 2019; 46: 441-449.

7. Bakke M, Henriksen T, Biernat HB, Dalager T, Møller E. Interdisciplinary recognizing and managing of drug-induced tardive oromandibular dystonia: two case reports. Clin Case Rep 2018; 6: 2150-2155

8. Beddis H, Pemberton M, Davies S. Sleep bruxism: an overview for clinicians. Br Dent J 2018; 225: 497-501.

9. Castrillon EE, Exposto FG. Sleep bruxism and pain. Dent Clin North Am 2018; 62: 657-663.

10. Chen YY, Fan HC, Tung MC, Chang YK. The association between Parkinson's disease and temporomandibular disorder. PLoS One 2019; 14: e0217763.

11. Cooper T, Smith L. Dental appliance therapy in pantothenate kinase-associated neurodegeneration: case report. Spec Care Dentist 2019; 39: 56-58.

12. Defazio G, Albanese A, Pellicciari R, et al. Expert recommendations for diagnosing cervical, oromandibular, and limb dystonia. Neurol Sci 2019; 40: 89-95.

13. Derry CP, Duncan JS, Berkovic SF. Paroxysmal motor disorders of sleep: the clinical spectrum and differentiation from epilepsy. Epilepsia 2006; 47: 1775-1791

14. Dufresne D, Dubovec K, So NK, Kotagal P. Ictal biting injuries in the epilepsy monitoring unit, a cohort study of incidence and semiological significance. Seizure 2019; 66: 39-41.
15. France K, Stoopler ET. The American Academy of Oral Medicine Clinical Practice Statement: oromandibular dystonia. Oral Surg Oral Med Oral Pathol Oral Radiol 2018; 125: 283-285.

16. Freitas ME, Ruiz-Lopez M, Dalmau J, et al. Seizures and movement disorders: phenomenology, diagnostic challenges and therapeutic approaches. J Neurol Neurosurg Psychiatry 2019; 90: 920-928.

17. Frisardi G, Iani C, Sau G, et al. A relationship between bruxism and orofacial-dystonia? A trigeminal electrophysiological approach in a case report of pineal cavernoma. Behav Brain Funct 2013; 9: 41

18. Jaramillo Jiménez E, Vargas García C, Rodríguez Márquez I, et al. Crisis psicógenas no epilépticas y crisis epilépticas: pistas para un diagnóstico diferencial. Hallazgos de unestudiocolombiano. Rev Neurol 2019; 69: 145.

19. Kato T, Montplaisir JY, Blanchet PJ, Lund JP, Lavigne GJ. Idiopathic myoclonus in the oromandibular region during sleep: a possible source of confusion in sleep bruxism diagnosis. Mov Disord 1999; 14: 865-871.

20. Lee SH, Park YB, Moon HS, et al. The role of rhFGF-2 soaked polymer membrane for enhancement of guided bone regeneration. J Biomater Sci Polym Ed 2018; 29: 825-843.

21. Lobbezoo F, Ahlberg J, Raphael KG, et al. International consensus on the assessment of bruxism: report of a work in progress. J Oral Rehabil 2018; 45: 837-844.

22. Lobbezoo F, Naeije M. Dental implications of some common movement disorders: a concise review. Arch Oral Biol 2007; 52 : 395-398.

23. Manfredini D, Ahlberg J, Wetselaar P, Svensson P, Lobbezoo F. The bruxism construct: from cut-off points to a continuum spectrum. J Oral Rehabil 2019; 46: 991-997.

24. Manfredini D, Lombardo L, Visentin A, Arreghini A, Siciliani G. Correlation between sleep-time masseter muscle activity and tooth wear: an electromyographic study. J Oral Facial Pain Headache 2019; 33: 199-204.

25. Mangiardi M, Alfano G. A clinical efficacy experience of Lacosamide on sleep quality in patients with nocturnal frontal lobe epilepsy (NFLE). Acta Biomed 2018; 89: 397-399.

26. Melo G, Duarte J, Pauletto P, et al. Bruxism: an umbrella review of systematic reviews. J Oral Rehabil 2019; 46: 666-690.

27. Muts EJ, van Pelt H, Edelhoff D, Krejci I, Cune M. Tooth wear: a systematic review of treatment options. J Prosthet Dent 2014; 112: 752-759.

28. Restrepo C, Manfredini D, Castrillon E, et al. Diagnostic accuracy of the use of parental-reported sleep bruxism in a polysomnographic study in children. Int J Paediatr Dent 2017; 27: 318-325.

29. Rodrigues NS, de Souza LC, Feitosa VP, et al. Effect of different conditioning/deproteinization protocols on the bond strength and degree of conversion of self-adhesive resin cements applied to dentin. Int J Adhes Adhes 2018; 81: 98-104.

30. Ryvlin P, Rheims S, Risse G. Nocturnal frontal lobe epilepsy. Epilepsia 2006; 47: 83-86.

31. Sarode G, Sarode S. Abfraction: a review. J Oral Maxillofac Pathol 2013; 17: 222

32. Shalash AS, Abushouk AI, Elsherbeny MY, Elrassas H, Kamel T. Refractory open jaw oromandibular tardive dystonia with a sensory trick, treated with botulinum toxin: a case report. Neurol India 2019; 67: 1110-1111.

33. Shellis RP, Addy M. The interactions between attrition, abrasion and erosion in tooth wear. Monogr Oral Sci 2014; 25: 32-45.

34. Smardz J, Martynowicz H, Michalek-Zrabkowska M, et al. Sleep bruxism and occurrence of temporomandibular disorders-related pain: a polysomnographic study. Front Neurol 2019; 10: 168.

35. Smardz J, Martynowicz H, Wojakowska A, Michalek-Zrabkowska M, Mazur G, Wieckiewicz M. Correlation between sleep bruxism, stress, and depression-a polysomnographic study. J Clin Med 2019; 8: 1344.

36. Tee TY, Khoo CS, Norlinah MI. Prominent oromandibular dystonia as levodopa-induced dyskinesia in idiopathic Parkinson's disease. Mov Disord Clin Pract 2019; 6: 330-331. 
37. Tinuper P, Provini F, Bisulli F, et al. Movement disorders in sleep: guidelines for differentiating epileptic from non-epileptic motor phenomena arising from sleep. Sleep Med Rev 2007; 11: 255-267.

38. Verhoeff MC, Lobbezoo F, Wetselaar P, Aarab G, Koutris M. Parkinson's disease, temporomandibular disorders and bruxism: A pilot study. J Oral Rehabil 2018; 45: 854-863.

39. Watts MW, Tan EK, Jankovic J. Bruxism and cranial-cervical dystonia: is there a relationship? Cranio 1999; 17: 196-201.

40. Wei Z, Du Y, Zhang J, Tai B, Du M, Jiang H. Prevalence and indicators of tooth wear among Chinese adults. PLoS One 2016; 11 e0162181.

41. Wetselaar P, Lobbezoo F. The tooth wear evaluation system: a modular clinical guideline for the diagnosis and management planning of worn dentitions. J Oral Rehabil 2016; 4: 69-80.

42. Yap AU, Chua AP. Sleep bruxism: current knowledge and contemporary management. J Conserv Dent 2016; 19: 383-389. 